\title{
TIPIC-A Newly Recognized Syndrome: Multimodality Imaging of a Rare Clinicoradiological Entity
}

\author{
Tanvi Modi ${ }^{1}$ Mitusha Verma ${ }^{1}$ Gauri Ahuja ${ }^{1}$ Neemish Kamat ${ }^{1}$ Deepak P Patkar ${ }^{1}$ \\ 1Department of Radiology, Dr. Balabhai Nanavati Hospital, Mumbai, \\ Maharashtra, India \\ Address for correspondence Tanvi Modi, A-701, Sorrento, Besant \\ Road, Santacruz-West, Mumbai - 400 054, Maharashtra, India \\ (e-mail: tanvimodi134@gmail.com).
}

Indian J Radiol Imaging 2021;31:488-491.

\begin{abstract}
Keywords

- carotidynia

- focal neck pain

- multimodality imaging

- TIPIC syndrome

Carotidynia is a controversial clinicopathological entity vastly described in Western literature as neck pain in the region of carotid bifurcation secondary to an underlying inflammatory etiology. Radiologically, this appears as perivascular inflammation and has recently been designated as transient perivascular inflammation of the carotid artery (TIPIC) syndrome. The authors of this report discuss the multimodality imaging features of a rare case of this disease in our country to familiarize radiologists with the imaging findings and to encourage the inclusion of TIPIC syndrome as a differential diagnosis for focal neck pain.
\end{abstract}

\section{Introduction}

Carotidynia, popularly known as Fay syndrome, ${ }^{1}$ is radiologically described as perivascular inflammation of the carotid sheath and carotid adventitia. ${ }^{2}$ Previously, it was a vague term used to describe neck pain associated with migraine and thought to be devoid of imaging features according to the International Headache Society. ${ }^{3,4}$ Recently, the radiological description of this condition has been given the name transient perivascular inflammation of the carotid artery (TIPIC) syndrome. ${ }^{5}$ A characteristic feature is focal eccentric enhancing thickening involving the carotid bifurcation without associated hemodynamic changes. An association with autoimmune diseases and upper airway infection was considered but neither has been proven in the past. This report showcases the imaging features of TIPIC syndrome in a patient with sudden onset of focal neck pain.

\section{Case Report}

A 35-year-old female presented to our department from an ENT referral. She presented with complaints of sudden onset left-sided neck pain that aggravated on neck movements. It was assumed to be secondary to upper airway infection;

published online July 28, 2021
DOI https://doi.org/ $10.1055 / \mathrm{s}-0041-1734353$ ISSN 0971-3026 however, there was no symptomatic relief after a course of antibiotics. On examination, the focal area of pain was seen to be extremely tender, with a tiny palpable lump like lesion associated with prominent pulsations. Of note, the patient did not have any complaints of migraine, Raynaud's phenomenon, tingling, and numbness of extremities.

Complete blood count and inflammatory markers such as erythrocyte sedimentation rate and C-reactive protein were found to be normal.

In significant past history, she was initiated on treatment for rheumatoid arthritis 10 years ago due to swelling of the interphalangeal joints of bilateral hands and bilateral sacroiliitis. However, she did not test positive for serological markers to confirm rheumatoid arthritis. She was initiated on disease-modifying antirheumatoid agents that were discontinued 3 years ago due to drug-induced acute hepatic failure.

The patient was referred for an ultrasound of the neck. A focal hypoechoic area was seen along the left common carotid artery at the level of bifurcation ( - Fig. 1), which was suspicious for dissection. No hemodynamic compromise was noted.

The patient was then sent for a magnetic resonance imaging (MRI) scan for further assessment. MRI revealed (c) 2021. Indian Radiological Association.

This is an open access article published by Thieme under the terms of the Creative Commons Attribution-NonDerivative-NonCommercial-License, permitting copying and reproduction so long as the original work is given appropriate credit. Contents may not be used for commercial purposes, or adapted, remixed, transformed or built upon. (https://creativecommons.org/licenses/by-nc-nd/4.0/).

Thieme Medical and Scientific Publishers Private Ltd. A-12, Second Floor, Sector -2, NOIDA -201301, India 
short segment near circumferential, ill-defined perivascular enhancing soft tissue and adventitial thickening at the level of left common carotid artery bifurcation ( - Fig. 2A), extending along the proximal portion of the left internal carotid artery (-Fig. 3A and B ). No significant luminal narrowing or loss of flow void was seen on the angiographic sequences (-Fig. 4A)

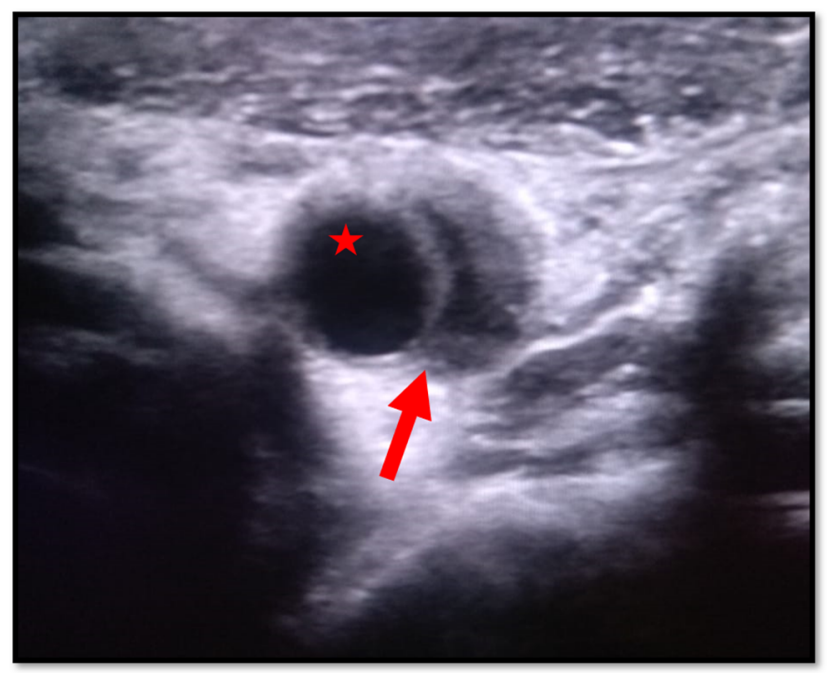

Fig. 1 Ultrasound image reveals iso to hypoechoic eccentric thickening (arrow) along left common carotid bifurcation. There is no luminal narrowing seen (asterisk).
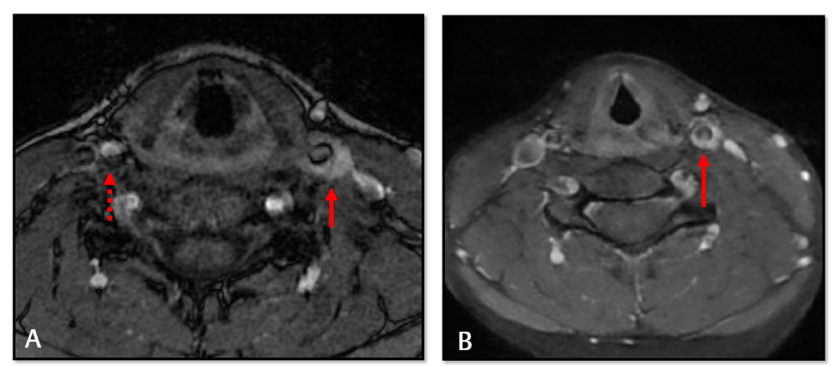

Fig. 2 (A) Axial T1-weighted postcontrast magnetic resonance imaging of the neck at the time of presentation reveals enhancement in perivascular region at the level of left carotid bifurcation (arrow). Note normal appearance of right carotid bifurcation (dotted arrow). (B) Postcontrast image at the time of follow-up reveals significant reduction in enhancing soft tissue (arrow).
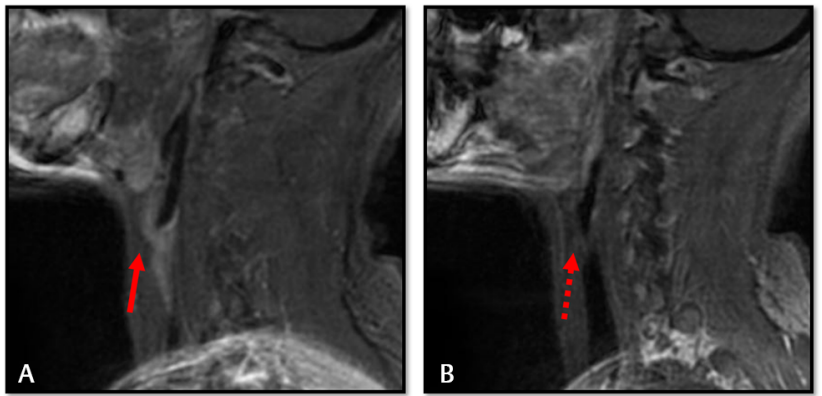

Fig. 3 (A) Sagittal T1-weighted postcontrast magnetic resonance imagings of the neck reveal the extension of the enhancing soft tissue along proximal internal carotid artery (arrow). (B) Note contralateral normal postcontrast appearance (dotted arrow).
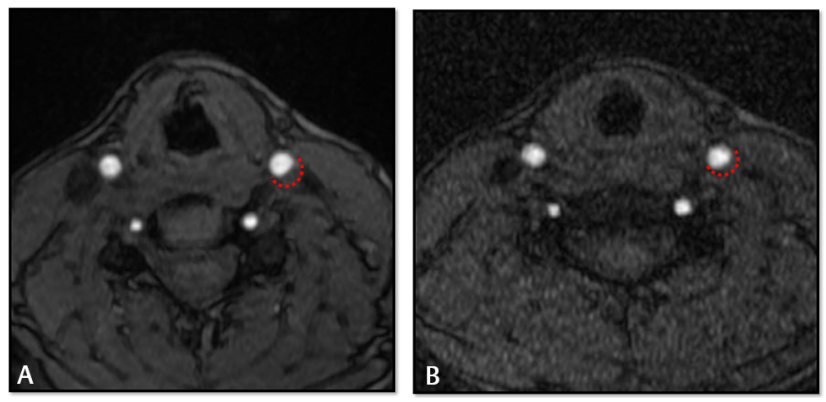

Fig. 4 (A) Axial magnetic resonance (MR) neck angiography image at the time of presentation reveals periluminal soft tissue (dotted line) with maintained luminal caliber. (B) Posttreatment MR angiographic image reveals reduction in the periluminal soft tissue (dotted line). Luminal caliber is maintained.

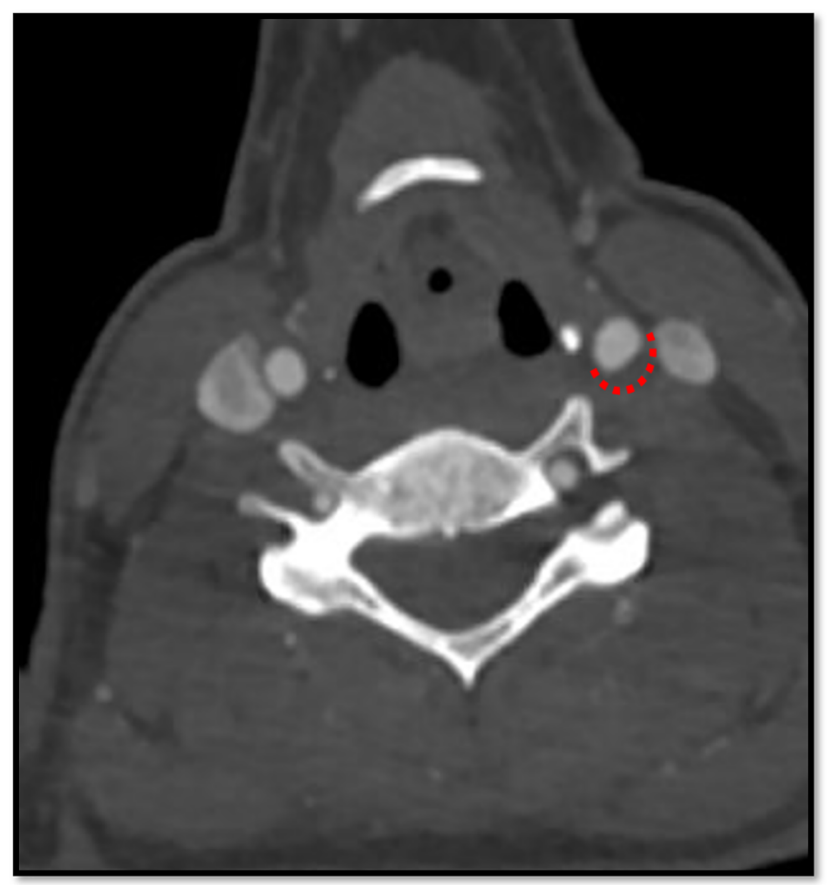

Fig. 5 Axial computed tomography angiographic image at the level of carotid bifurcation reveals perivascular soft tissue involving the left common carotid artery (dotted line).

After dissection was ruled out, the patient was advised regarding the benign nature of the condition and given a course of injectable steroids. Further, workup to rule out vasculitis was done by performing a computed tomography (CT) aortogram that revealed focal asymmetric thickening along the left common carotid artery at its bifurcation (-Fig. 5). No associated luminal narrowing was seen. On the basis of the spectrum of imaging findings with associated clinical features, a diagnosis of carotidynia (TIPIC syndrome) was made.

The patient again presented with complaints of pain a monthlater, when a whole-body fluorodeoxyglucose-positron emission tomography-computed tomography (FDG-PET-CT) was performed to rule out vasculitis and look for a source of infection or inflammation. No abnormal tracer uptake was noted on whole-body FDG-PET-CT ( - Fig. 6). 


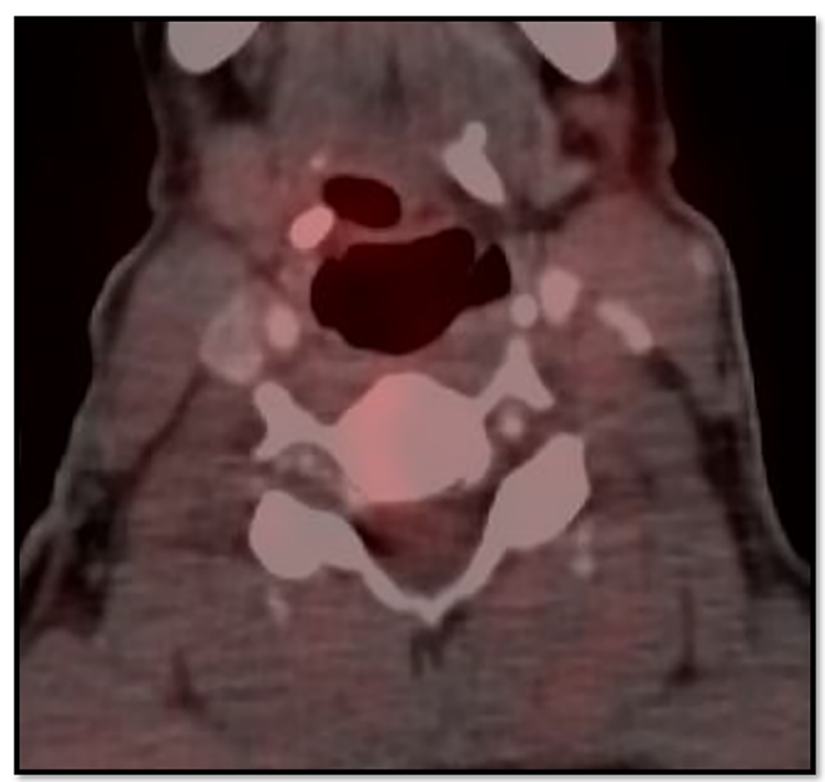

Fig. 6 Fused positron emission tomography-computed tomographic image reveals no abnormal tracer uptake at the level of the left carotid bifurcation.

A 6-month follow-up MRI was performed that revealed a significant reduction in thickness and extent of perivascular soft tissue ( - Fig. 2B and B ).

\section{Discussion}

There is some ambiguity regarding the definition of this disease condition with some authors refuting the existence of carotidynia. ${ }^{6}$ Burton et $\mathrm{al}^{7}$ published a case series of five patients demonstrating the MRI findings in patients with carotidynia. MR angiography and postcontrast MR clearly depict the involvement of adventitia by enhancing soft tissue with sparing of the lumen. MR also excludes dissection, intramural hematoma by the absence of Tl hyperintense signal within the wall. Cross-sectional imaging also rules out other vascular conditions that may cause unilateral neck pain such as arterial dissection, aneurysms, and fibromuscular dysplasia.

The ultrasonographic findings of Santarosa et $\mathrm{al}^{8}$ showed the wall thickening to be isoechoic to surrounding muscles with no luminal narrowing. Doppler evaluation showed no significant hemodynamic compromise. Amaravadi et $\mathrm{al}^{9}$ performed a study on a series of patients with carotidynia using PET-CT. The study revealed increased tracer uptake in the pericarotid region, likely due to glucose hypermetabolism, confirming underlying inflammatory pathology.

Regardless of the imaging modality (ultrasound, CT, or MRI), the typical features of TIPIC syndrome include involvement of distal common carotid artery, bulb and proximal internal carotid artery, mild luminal narrowing, eccentric perivascular and adventitial thickening, enhancement (more so on MRI), and transient fatty plaque during healing. ${ }^{10}$

Histopathological confirmation is rarely performed due to the potential hazards of taking a tissue sample near the carotid vessels. A one of its kind histopathological study by Upton et $\mathrm{al}^{2}$ demonstrated nonspecific inflammatory changes within the perivascular tissue sample. However, this cannot be performed routinely; hence; imaging is the mainstay for diagnosis.

Our case confirms the ultrasound, CT, and MRI findings of previous studies. However, increased uptake on PET was not seen. Few studies have shown the disappearance of imaging abnormalities; however, this was not seen in our study. The amount of perivascular soft tissue subsided in thickness and extent; however, it did not completely resolve. Similar findings were documented by Lecler et al. ${ }^{5}$

Clinically, our patient presented with sudden onset of symptoms. While most patients present with dull aching indolent pain, occasionally there may be patients presenting with acute pain. ${ }^{8}$ Few studies have reported the presence of an underlying autoimmune disease in patients presenting with carotidynia. One such study was conducted by Lecler et al, ${ }^{5}$ where 8 out of 47 patients in the study had an underlying autoimmune disease. The inflammatory nature of the disease is confirmed by the resolution of symptoms and reduction in perivascular soft tissue after a course of steroids.

In conclusion, this study depicts the imaging findings of this rare entity using different modalities. The symptomatic relief and improvement on MRI after a course of anti-inflammatory medication confirm the inflammatory nature of the disease. While the pathogenesis of this entity is unknown, the possibility of an association between autoimmune diseases and carotidynia has been raised by a few studies; however, dedicated investigation in a larger study population is warranted.

\section{Declaration of Patient Consent}

The authors certify that they have obtained all appropriate patient consent forms. In the form, the patient(s) has/ have given his/her/their consent for his/her/their images and other clinical information to be reported in the journal. The patients understand that their names and initials will not be published and due efforts will be made to conceal their identity, but anonymity cannot be guaranteed.

\section{Financial Support and Sponsorship}

Nil.

\section{Conflicts of Interest}

There are no conflicts of interest.

\section{References}

1 Fay T. Atypical neuralgia. Arch Neurol Psychiatry 1927;18:309-315

2 Upton PD, Smith JG, Charnock DR. Histologic confirmation of carotidynia. Otolaryngol Head Neck Surg 2003;129(4):443-444

3 Headache Classification Committee of the International Headache Society. Classification and diagnostic criteria for headache disorders, cranial neuralgias and facial pain. Cephalalgia 1988;8(Suppl 7):1-96

4 Murray TJ. Carotidynia: a cause of neck and face pain. Can Med Assoc J 1979;120(4):441-443 
5 Lecler A, Obadia M, Savatovsky J, et al. TIPIC syndrome: beyond the myth of carotidynia, a new distinct unclassified entity. AJNR Am J Neuroradiol 2017;38(7):1391-1398

6 Biousse V, Bousser MG. The myth of carotidynia. Neurology 1994;44(6):993-995

7 Burton BS, Syms MJ, Petermann GW, Burgess LP. MR imaging of patients with carotidynia. AJNR Am J Neuroradiol 2000;21(4):766-769
8 Santarosa C, Stefanelli S, Sztajzel R, Mundada P, Becker M. Carotidynia: a rare diagnosis for unilateral neck pain revealed by cross-sectional imaging. Case Rep Radiol 2017;2017:7086854

9 Amaravadi RR, Behr SC, Kousoubris PD, Raja S. [18F] Fluorodeoxyglucose positron-emission tomography-CT imagingofcarotidynia.AJNRAmJNeuroradiol2008;29(6):1197-1199

10 Woo JK, Jhamb A, Heran MK, Hurley M, Graeb D. Resolution of existing intimal plaque in a patient with carotidynia. AJNR Am J Neuroradiol 2008;29(4):732-733 